\title{
EIGENFUNCTION EXPANSIONS ASSOCIATED WITH THE LAPLACIAN FOR CERTAIN DOMAINS WITH INFINITE BOUNDARIES. II. APPLICATIONS TO SCATTERING THEORY $\left({ }^{1}\right)$
}

\author{
BY \\ CHARLES IRWIN GOLDSTEIN
}

1. Introduction. In this paper we shall employ the distorted plane waves (generalized eigenfunctions) $w_{n}^{ \pm}(x ; \xi)$ constructed in a preceding paper $[1]\left({ }^{2}\right)$ to investigate the problem of scattering in a perturbed infinite cylinder $\Omega$. We assume that the reader is familiar with the notation used in I.

Generalized eigenfunctions were first used to construct a scattering operator by Ikebe [2]. He studied the operators $-\Delta$ and $-\Delta+q(x)$ acting on $L_{2}\left(R^{N}\right)$, where $q(x)$ is a potential function. This method was also used to construct a scattering operator for the wave equation in the exterior of a bounded obstacle by Shenk [5]. The exterior problem has been treated by Lax and Phillips [4], using a different approach. They constructed the scattering operator with the aid of certain abstract representation theorems. They then applied their theory to obtain a complete set of generalized eigenfunctions of the reduced wave equation in an exterior domain. The methods of [4] are not applicable in their present form to our problem.

We begin in $\S 2$ by defining certain Hilbert spaces $\mathscr{H}_{0}$ and $\mathscr{H}_{1}$ of initial data for the wave equation with zero boundary conditions in $S$ and $\Omega$, respectively. (It may easily be shown that all of the arguments in this paper carry over to the case of a semi-infinite cylinder as well as to the case of the boundary conditions discussed in I.) In Theorem 2.1 we establish the existence of a group of unitary transformations $\mathscr{U}_{0}(t)[\mathscr{U}(t)]$ acting on $\mathscr{H}_{0}[\mathscr{H}]$.

In $\S 3$, we construct spectral representations for the operators $\mathscr{U}_{0}(t)[\mathscr{U}(t)]$. These spectral representations are expressed in terms of the functions $w_{n}^{ \pm}(x ; \xi)$. In $\S 4$, we define the wave operators $\mathscr{W}^{ \pm}$. We prove that the operators $\mathscr{W}^{ \pm}$exist as unitary transformations. This follows from Theorem 4.1.

We discuss the scattering operator $\mathscr{S}=\left(\mathscr{W}^{+}\right)^{-1} \mathscr{W}^{-}$in $\S 5$. An immediate consequence of the existence of the scattering operator is a local energy decay theorem

Received by the editors August 6, 1967.

( ${ }^{1}$ Part of this work was performed under the auspices of the U.S. Atomic Energy Commission Contract AT(30-1)-1480 at the Courant Institute of Mathematical Sciences, New York University, New York.

${ }^{(2)}[1]$ will be referred to as I. 
for the solutions $\mu^{0}(x, t)$ of the wave equation in $S$ with finite energy, which vanish on $\dot{S}$. It is shown that

$$
\int_{B}\left[\left|\nabla \mu^{0}(x, t)\right|^{2}+\left|\mu_{t}^{0}(x, t)\right|^{2}\right] d x \rightarrow 0 \quad \text { as } t \rightarrow \pm \infty
$$

for each bounded subset $B$ of $S$. No information is given, however, concerning the rate of decay.

Finally, in $\S 6$ we extend the arguments of $\S 4$ to establish the existence, unitarity, and invariance of the wave operators $W^{ \pm}\left(\varphi\left(A^{1 / 2}\right), \varphi\left(A_{0}^{1 / 2}\right)\right)$ for a wide class of real-valued functions $\varphi$. A result of this kind was obtained by Kato [3] for the case in which $A_{0}$ and $A$ are selfadjoint operators whose difference is of trace class. Shenk [6] treated the case in which $A_{0}=-\Delta$ on $L_{2}\left(R^{N}\right)$, and $A=-\Delta$ on $L_{2}(\Omega)$, $\Omega$ being a domain with finite, smooth boundary.

2. The unitary groups. Suppose $S$ is an infinite cylinder in $R^{N}(N \geqq 2)$ and $\Omega \subseteq S$ is the perturbed infinite cylinder defined in I. For simplicity we assume that the selfadjoint operator $A$ has no point eigenvalues. The case in which eigenvalues are present will be discussed in $\S 4$. We begin by defining Hilbert spaces, $\mathscr{H}_{0}$ and $\mathscr{H}$, of initial data in $S$ and $\Omega$, respectively. Given $\varphi_{(1)}(x)$ in $C_{0}^{\infty}(\Omega)$ and $\varphi_{(2)}(x)$ in $C_{0}^{\infty}(\Omega)$, set $\varphi=\left[\varphi_{(1)}, \varphi_{(2)}\right]$ and

$$
\|\varphi\|_{\mathscr{H}}^{2}=\int_{\Omega}\left[\left|\nabla \varphi_{(1)}(x)\right|^{2}+\left|\varphi_{(2)}(x)\right|^{2}\right] d x .
$$

Define $\mathscr{H}$ to be the completion of $C_{0}^{\infty}(\Omega) \oplus C_{0}^{\infty}(\Omega)$ under the norm, \|\|$_{\mathscr{H}}$. We define a Hilbert space $\mathscr{H}_{0}$ similarly.

It follows from the definitions that $\mathscr{H}_{0}=B_{0} \oplus L_{2}(S)$ and $\mathscr{H}=B \oplus L_{2}(\Omega)$, where $B_{0}[B]$ is the completion of $C_{0}^{\infty}(S)\left[C_{0}^{\infty}(\Omega)\right]$ under the norm given by $\int_{S}|\nabla \varphi(x)|^{2} d x$ $\left[\int_{\Omega}|\nabla \varphi(x)|^{2} d x\right]$. It is easy to see that $B_{0}=\dot{H}_{1}(S)$ and $B=\dot{H}_{1}(\Omega)$. This follows from the estimate

$$
\|\varphi\|_{0_{(S)}}\left[\|\varphi\|_{0_{(\Omega)}}\right] \leqq C\|\nabla \varphi\|_{0_{(S)}}\left[C\|\nabla \varphi\|_{0_{(\Omega)}}\right]
$$

where the constant $C$ is independent of the function $\varphi(x)$ in $C_{0}^{\infty}(S)\left[C_{0}^{\infty}(\Omega)\right]$. (2.1) is clearly equivalent to the fact that 0 is not in the spectrum of $A_{0}[A]$.

We next establish the existence of solutions of the initial value problem for the wave equation in $\Omega$ with zero boundary values on $\dot{\Omega}$. (The same proof goes through for the homogeneous boundary conditions considered in I.) This will be accomplished by means of a separation of variables in the representation space

$$
H=\bigoplus_{n=1}^{\infty} L_{2}\left(-\infty, \infty ; d \rho_{n}(\xi)\right) \quad \text { where } d \rho_{n}(\xi)=d \xi
$$

THEOREM 2.1. Suppose $\varphi_{(1)}(x) \in C_{0}^{\infty}(\Omega)$ and $\varphi_{(2)}(x) \in C_{0}^{\infty}(\Omega)$. Then

(a) There exists a function $\mu^{+}(x, t)$ defined in $\Omega \times R^{1}$, satisfying the conditions

$$
\begin{gathered}
\partial^{k} \mu^{+} / \partial t^{k} \in D(A), \quad \text { for } k=0,1, \ldots, \\
\Delta \mu^{+}=\mu_{t t}^{+}, \quad \text { and } \quad \mu^{+}(x, 0)=\varphi_{(1)}(x), \quad \mu_{t}^{+}(x, 0)=\varphi_{(2)}(x) .
\end{gathered}
$$


(b) There exist functions $\alpha_{n}^{+}(\xi)$ and $\beta_{n}^{+}(\xi)$ such that

$$
\left(\mu^{+}(\cdot, t)\right)_{n}^{+\uparrow}(\xi)=\alpha_{n}^{+}(\xi) \exp \left[i\left(\xi^{2}+\nu_{n}\right)^{1 / 2} t\right]+\beta_{n}^{+}(\xi) \exp \left[-i\left(\xi^{2}+\nu_{n}\right)^{1 / 2} t\right] .
$$

Also

$$
\left(\frac{\partial^{k} \mu^{+}}{\partial t^{k}}(\cdot, t)\right)_{n}^{+\uparrow}(\xi)=\frac{\partial^{k}}{\partial t^{k}}\left(\mu^{+}(\cdot, t)\right)_{n}^{+\wedge}(\xi)
$$

(c)

$$
\left|\mu^{+}(\cdot, t)\right|_{1_{(\Omega)}^{2}}^{2}=2 \sum_{n=1}^{\infty} \int_{-\infty}^{\infty}\left(\xi^{2}+\nu_{n}\right)\left[\left|\alpha_{n}^{+}(\xi)\right|^{2}+\left|\beta_{n}^{+}(\xi)\right|^{2}\right] d \xi
$$

where we define

$$
\left|\mu^{+}(\cdot, t)\right|_{1_{(\Omega)}^{2}}^{2}=\int_{\Omega}\left[\left|\nabla \mu^{+}(x, t)\right|^{2}+\left|\mu_{t}(x, t)\right|^{2}\right] d x .
$$

Therefore energy is conserved.

(d) We may replace ${ }^{+\wedge}$ by ${ }^{-\wedge}$ to obtain a solution $\mu^{-}(x, t)$. We have

$$
\mu^{+}(x, t)=\mu^{-}(x, t) .
$$

Proof. (a) Set

$$
\begin{aligned}
\mu^{+}(x, t)=\sum_{n=1}^{\infty} \int_{-\infty}^{\infty} w_{n}^{+}(x ; \xi)\left[\alpha_{n}^{+}(\xi)\right. & \exp \left[i\left(\xi^{2}+\nu_{n}\right)^{1 / 2} t\right] \\
& \left.+\beta_{n}^{+}(\xi) \exp \left[-i\left(\xi^{2}+\nu_{n}\right)^{1 / 2} t\right]\right] d \xi
\end{aligned}
$$

where

$$
\begin{aligned}
& 2 i\left(\xi^{2}+\nu_{n}\right)^{1 / 2} \alpha_{n}^{+}(\xi)=i\left(\xi^{2}+\nu_{n}\right)^{1 / 2} \varphi_{(1)_{n}}^{+}(\xi)+\varphi_{(2)_{n}}^{+}(\xi), \\
& 2 i\left(\xi^{2}+\nu_{n}\right)^{1 / 2} \beta_{n}^{+}(\xi)=i\left(\xi^{2}+\nu_{n}\right)^{1 / 2} \varphi_{(1)_{n}}^{+}(\xi)-\varphi_{(2)_{n}}^{+}(\xi) .
\end{aligned}
$$

By Theorem 5.1 in I, we have

$$
\left(\mu^{+}\right)_{n}^{+}(\xi)=\alpha_{n}^{+}(\xi) \exp \left[i\left(\xi^{2}+\nu_{n}\right)^{1 / 2} t\right]+\beta_{n}^{+}(\xi) \exp \left[-i\left(\xi^{2}+\nu_{n}\right)^{1 / 2} t\right] .
$$

Since $\sum_{n=1}^{\infty} \int_{-\infty}^{\infty}\left|\xi^{2}+\nu_{n}\right|^{M}\left|\varphi_{(j)_{n}}^{+}(\xi)\right|^{2} d \xi<\infty$ for $j=1,2$, and $M=1,2, \ldots$, we conclude from (2.3), (2.4), and Theorem 4.1 of I that $\mu^{+} \in D\left(A^{M}\right)$ for $M=1,2, \ldots$

Set

$$
\begin{aligned}
& \mu_{\kappa}^{+}(x, t)=\sum_{n=1}^{\infty} \int_{-\infty}^{\infty} w_{n}^{+}(x ; \xi) {\left[i^{\kappa}\left(\xi^{2}+\nu_{n}\right)^{\kappa / 2} \alpha_{n}^{+}(\xi) \exp \left\{i\left(\xi^{2}+\nu_{n}\right)^{1 / 2} t\right\}\right.} \\
&\left.+(-i)^{\kappa}\left(\xi^{2}+\nu_{n}\right)^{\kappa / 2} \beta_{n}^{+}(\xi) \exp \left\{-i\left(\xi^{2}+\nu_{n}\right)^{1 / 2} t\right\}\right] d \xi,
\end{aligned}
$$

$\kappa=0,1, \ldots$. It follows readily that the derivatives $\partial^{\kappa} \mu^{+}(x, t) / \partial t^{\kappa}$ (considered in the sense of distributions in $\left.\Omega \times R^{1}\right)$ are equal to $\mu_{\kappa}^{+}(x, t), \kappa=0,1, \ldots$ Now

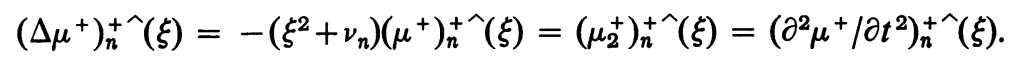

Thus $\Delta \mu^{+}=\partial^{2} \mu^{+} / \partial t^{2}$. From the definitions of $\mu^{+}$and $\mu_{1}^{+}$, it follows that $\mu^{+}(x, 0)$ $=\varphi_{1}(x)$ and $\partial \mu^{+}(x, 0) / \partial t=\varphi_{2}(x)$. This completes the proof of part (a). 
(b) This follows immediately from (2.4), (2.5), and the results of I.

(c) Integrating by parts and using the boundary condition and the spectral representation, we have

$$
\begin{aligned}
\left|\mu^{+}(\cdot, t)\right|_{1_{(\Omega)}}^{2}=\int_{\Omega}\left[\left|\nabla \mu^{+}(x, t)\right|^{2}+\left|\frac{\partial \mu^{+}(x, t)}{\partial t}\right|^{2}\right] d x \\
=\int_{\Omega}\left[\mu^{+}(x, t)\left(-\Delta \bar{\mu}^{+}(x, t)\right)+\left|\mu_{1}^{+}(x, t)\right|^{2}\right] d x \\
=\sum_{n=1}^{\infty} \int_{-\infty}^{\infty}\left(\xi^{2}+\nu_{n}\right)\left|\alpha_{n}^{+}(\xi) \exp \left[i\left(\xi^{2}+\nu_{n}\right)^{1 / 2} t\right]+\beta_{n}^{+}(\xi) \exp \left[-i\left(\xi^{2}+v_{n}\right)^{1 / 2} t\right]\right|^{2} \\
\quad+\left|i\left(\xi^{2}+v_{n}\right)^{1 / 2} \alpha_{n}^{+}(\xi) \exp \left[i\left(\xi^{2}+v_{n}\right)^{1 / 2} t\right]-i\left(\xi^{2}+v_{n}\right)^{1 / 2} \beta_{n}^{+}(\xi) \exp \left[-i\left(\xi+v_{n}\right)^{1 / 2} t\right]\right|^{2} \\
=2 \sum_{n=1}^{\infty} \int_{-\infty}^{\infty}\left[\left|\alpha_{n}^{+}(\xi)\right|^{2}+\left|\beta_{n}^{+}(\xi)\right|^{2}\right]\left(\xi^{2}+\nu_{n}\right) d \xi .
\end{aligned}
$$

This proves part (c).

(d) All of the preceding arguments go through with ${ }^{+\wedge}$ replaced by ${ }^{-\wedge}$. We thus have a function $\mu^{-}(x, t)$ satisfying the conditions

(i) $\left(\mu^{-}\right)^{-\wedge}=\alpha_{n}^{-}(\xi) \exp \left[i\left(\xi^{2}+\nu_{n}\right)^{1 / 2} t\right]+\beta_{n}^{-}(\xi) \exp \left[-i\left(\xi^{2}+\nu_{n}\right)^{1 / 2} t\right]$,

(ii) $\Delta \mu^{-}=\mu_{t t}^{-}$,

(iii) $\mu^{-}(x, 0)=\varphi_{1}(x)$,

(iv) $\mu_{t}^{-}(x, 0)=\varphi_{2}(x)$, and

(v) $\partial^{x} \mu^{-} / \partial t^{x} \in D(A)$.

We show that $\mu^{+}(x, t)=\mu^{-}(x, t)$. Set $v(x, t)=\mu^{+}(x, t)-\mu^{-}(x, t)$. Note that

$$
\|v(\cdot, t)\|_{1_{(\Omega)}}+\left\|v_{t}(\cdot, t)\right\|_{1_{(\Omega)}}+\|\Delta v(\cdot, t)\|_{0_{(\Omega)}} \leqq C_{T}
$$

for $|t| \leqq T$. Now, since $v(x, t)$ satisfies conditions (ii) and (v), we may apply Green's formula and estimate (2.6) (to justify interchanging the order of integrations) to obtain

$$
\begin{aligned}
\int_{\Omega}\left[|\nabla v(x, t)|^{2}+\left|v_{t}(x, t)\right|^{2}\right] d x \\
\quad=\int_{\Omega} d x \int_{0}^{t} \frac{d}{d t^{\prime}}\left[\left|\nabla v\left(x, t^{\prime}\right)\right|^{2}+\left|v_{t^{\prime}}\left(x, t^{\prime}\right)\right|^{2}\right] d t^{\prime}+\int_{\Omega}\left[|\nabla v(x, 0)|^{2}+\left|v_{t}(x, 0)\right|^{2}\right] d x \\
\quad=2 \operatorname{Re} \int_{\Omega} \int_{0}^{t}\left[\nabla v_{t}\left(x, t^{\prime}\right) \nabla \bar{v}\left(x, t^{\prime}\right)+v_{t}\left(x, t^{\prime}\right) \Delta \bar{v}\left(x, t^{\prime}\right)\right] d t^{\prime} d x \\
\quad=2 \operatorname{Re} \int_{0}^{t} \int_{\Omega}\left[\nabla v_{t}\left(x, t^{\prime}\right) \nabla \bar{v}\left(x, t^{\prime}\right)+v_{t}\left(x, t^{\prime}\right) \Delta \bar{v}\left(x, t^{\prime}\right)\right] d x d t^{\prime} \\
\quad=2 \operatorname{Re} \int_{0}^{t} \int_{\Omega}\left[-v_{t}\left(x, t^{\prime}\right) \Delta \bar{v}\left(x, t^{\prime}\right)+v_{t}\left(x, t^{\prime}\right) \Delta \bar{v}\left(x, t^{\prime}\right)\right] d x d t^{\prime} \\
=0 .
\end{aligned}
$$


This shows that $\mu^{+}(x, t)=\mu^{-}(x, t)$. The proof of Theorem 2.1 is thus complete. Q.E.D.

We denote $\mu^{+}(x, t)=\mu^{-}(x, t)$ by $\mu(x, t)$. We now define a group of unitary mappings $\mathscr{U}(t)$ from $\mathscr{H}$ onto $\mathscr{H}$. Suppose $\varphi_{j}(x) \in C_{0}^{\infty}(\Omega), j=1,2$, and $\varphi=\left[\varphi_{1}, \varphi_{2}\right]$. Set

$$
\mathscr{U}(t) \varphi=\left[\mu(\cdot, t), \mu_{t}(\cdot, t)\right]
$$

By the conservation of energy $\mathscr{U}(t)$ is isometric. Extend $\mathscr{U}(t)$ to all of $\mathscr{H}$ by continuity. It is easily seen that they form a strongly continuous group of unitary transformations from $\mathscr{H}$ onto $\mathscr{H}$, i.e.

$$
\begin{aligned}
\mathscr{U}(t): \mathscr{H} & \stackrel{\text { onto }}{\longrightarrow} \mathscr{H} \\
(\mathscr{U}(t))^{-1} & =(\mathscr{U}(t))^{*}=\mathscr{U}(-t), \quad-\infty<t<\infty \\
\mathscr{U}\left(t_{1}\right) \mathscr{U}\left(t_{2}\right) & =\mathscr{U}\left(t_{1}+t_{2}\right), \quad-\infty<t_{1}, t_{2}<\infty \\
\lim _{t \rightarrow 0}\|\mathscr{U}(t) f-f\|_{\mathscr{H}} & =0 \quad \text { for each } f \text { in } \mathscr{H} .
\end{aligned}
$$

Similarly if $\varphi_{j}(x) \in C_{0}^{\infty}(S), j=1,2$, and $\varphi=\left[\varphi_{1}, \varphi_{2}\right]$, we set $\mathscr{U}_{0}(t) \varphi=\left[\mu^{0}(\cdot, t)\right.$, $\left.\mu_{t}^{0}(\cdot, t)\right]$, where the function $\mu^{0}(x, t)$ may be constructed in the same manner as $\mu(x, t)$ with $\Omega$ replaced by $S$. Again $\mathscr{U}_{0}(t)$ may be extended to form a strongly continuous group of unitary transformations from $\mathscr{H}_{0}$ onto $\mathscr{H}_{0}$.

3. The spectral representations. In this section we shall construct spectral representations, $\mathscr{T}^{0}$ and $\mathscr{T}^{ \pm}$, for the unitary groups $\mathscr{U}_{0}(t)$ and $\mathscr{U}(t)$, respectively. The range of these spectral representations will be the Hilbert space $H^{2}=H \oplus H$.

Suppose $f=\left[f_{1}, f_{2}\right] \in \mathscr{H}$. Set

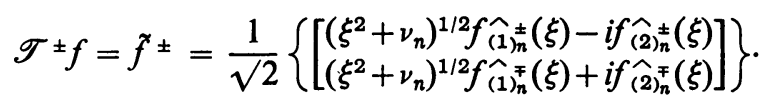

$\mathscr{T}^{ \pm} f \in H^{2}$. Similarly, for $g=\left[g_{1}, g_{2}\right]$ in $\mathscr{H}_{0}$, set

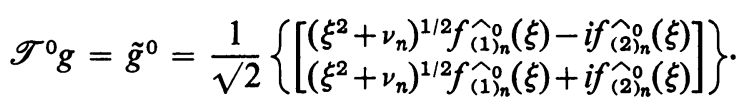

THEOREM 3.1. (a) $\mathscr{T}^{ \pm}$is a unitary mapping from $\mathscr{H}$ onto $H^{2}$.

(b) For each $f$ in $\mathscr{H}$, we have

$$
\mathscr{T}^{ \pm}(\mathscr{U}(t) f)=\left[\exp \left\{i\left(\xi^{2}+v_{n}\right)^{1 / 2} t\right\} F_{(1)_{n}}^{ \pm}(\xi), \exp \left\{-i\left(\xi^{2}+v_{n}\right)^{1 / 2} t\right\} F_{(2)_{n}}^{ \pm}(\xi)\right],
$$

where $\mathscr{T}^{ \pm} f=\left[F_{(1)_{n}}^{ \pm}(\xi), F_{(2)_{n}}^{ \pm}(\xi)\right]$. 
Proof. (a) We shall carry out the proof for the transformation $\mathscr{T}^{+}$. We first show that $\mathscr{T}^{+}$is isometric on a dense subspace of $\mathscr{H}$. Suppose $\varphi=\left[\varphi_{1}, \varphi_{2}\right] \in$ $\left(C_{0}^{\infty}(\Omega)\right) \oplus\left(C_{0}^{\infty}(\Omega)\right)$.

$$
\begin{aligned}
\|\varphi\|_{\mathscr{H}}^{2}= & \int_{\Omega}\left[\left|\nabla \varphi_{1}\right|^{2}+\left|\varphi_{2}\right|^{2}\right] d x \\
= & \frac{1}{2} \sum_{n=1}^{\infty} \int_{-\infty}^{\infty}\left[\left(\xi^{2}+\nu_{n}\right)\left|\varphi_{(1)_{n}}^{+}(\xi)\right|^{2}+\left|\varphi_{(2)_{n}}^{+}(\xi)\right|^{2}\right] d \xi \\
& +\frac{1}{2} \sum_{n=1}^{\infty} \int_{-\infty}^{\infty}\left[\left(\xi^{2}+\nu_{n}\right)\left|\varphi_{(1)_{n}}^{-} \hat{(\xi)}(\xi)\right|^{2}+\left|\varphi_{(2)_{n}}^{-\hat{n}}(\xi)\right|^{2}\right] d \xi
\end{aligned}
$$

using Theorem 4.1 of $\mathrm{I}$.

$$
\begin{aligned}
& \left\|\mathscr{T}^{+} \varphi\right\|_{H^{2}}^{2}=\frac{1}{2} \sum_{n=1}^{\infty} \int_{-\infty}^{\infty}\left|\left(\xi^{2}+\nu_{n}\right)^{1 / 2} \varphi_{(1)_{n}}^{+}(\xi)+i \varphi_{(2)_{n}}^{+}(\xi)\right|^{2} d \xi \\
& +\frac{1}{2} \sum_{n=1}^{\infty} \int_{-\infty}^{\infty}\left|\left(\xi^{2}+\nu_{n}\right)^{1 / 2} \varphi_{(1)_{n}}^{-}(\xi)-i \varphi_{(2)_{n}}^{-\hat{(\xi)}}\right|^{2} d \xi \\
& =\frac{1}{2} \sum_{n=1}^{\infty} \int_{-\infty}^{\infty}\left(\xi^{2}+\nu_{n}\right)\left|\varphi_{(1)_{n}}^{+}(\xi)\right|^{2} d \xi+\frac{1}{2} \sum_{n=1}^{\infty} \int_{-\infty}^{\infty}\left|\varphi_{(2)_{n}}^{+}(\xi)\right|^{2} d \xi \\
& +\frac{1}{2} \sum_{n=1}^{\infty} \int_{-\infty}^{\infty}\left(\xi^{2}+\nu_{n}\right)\left|\varphi_{(1)_{n}}^{-}(\xi)\right|^{2} d \xi+\frac{1}{2} \sum_{n=1}^{\infty} \int_{-\infty}^{\infty}\left|\varphi_{(2)_{n}}^{-\widehat{l}}(\xi)\right|^{2} d \xi \\
& +\frac{1}{2} \sum_{n=1}^{\infty} \int_{-\infty}^{\infty}\left(\left[i\left(\xi^{2}+\nu_{n}\right)^{1 / 2} \varphi_{(1)_{n}}^{+}(\xi)\left[\varphi_{(2) \hat{n}}^{+}(\xi)\right]^{*}-i\left(\xi^{2}+\nu_{n}\right)^{1 / 2}\left[\varphi_{(1)_{n}}^{+}(\xi)\right]^{*} \varphi_{(2)_{n}}^{+}(\xi)\right]\right) d \xi \\
& -\frac{1}{2} \sum_{n=1}^{\infty} \int_{-\infty}^{\infty}\left(\left[i\left(\xi^{2}+\nu_{n}\right)^{1 / 2} \varphi_{(1)_{n}}^{-}(\xi)\left[\varphi_{(2)_{n}}^{-}(\xi)\right]^{*}-i\left(\xi^{2}+\nu_{n}\right)^{1 / 2}\left[\varphi_{(1)_{n}}^{-}(\xi)\right]^{*} \varphi_{(2)_{n}}^{-}(\xi)\right]\right) d \xi \\
& =\|\varphi\|_{\mathscr{H}}^{2}
\end{aligned}
$$

$$
\begin{aligned}
\sum_{n=1}^{\infty} \int_{-\infty}^{\infty}\left(\xi^{2}+\nu_{n}\right)^{1 / 2} \varphi_{(1)_{n}}^{+\hat{n}}(\xi)\left[\varphi_{(2)_{n}}^{+\hat{\gamma}}(\xi)\right]^{*} d \xi & =\sum_{n=1}^{\infty} \int_{-\infty}^{\infty}\left(\xi^{2}+\nu_{n}\right)^{1 / 2} \varphi_{(1)_{n}}^{-\hat{n}}(\xi)\left[\varphi_{(2)_{n}}^{-}(\xi)\right]^{*} d \xi \\
& =\left(A^{1 / 2} \varphi_{(1)}, \varphi_{(2)}\right)_{L_{2}(\Omega)}
\end{aligned}
$$

again employing the expansion Theorem 4.1 of I. Note that * denotes the complex conjugate. Thus we may extend $\mathscr{T}^{+}$isometrically to all of $\mathscr{H}$.

We next show that $\mathscr{T}^{+}$is onto. Observe that $H^{2}$ may be expressed as the direct sum of two subspaces $\mathscr{S}^{E}$ and $\mathscr{S}^{\circ}$, where $\mathscr{S}^{E}$ consists of "even" elements of the form $F=\left\{\left[F_{n}(\xi), T^{-}\left(T^{+}\right)^{-1} F_{n}(\xi)\right]\right\}$ and $\mathscr{S}^{\circ}$ consists of "odd" elements of the form $G=\left\{\left[G_{n}(\xi),-T^{-}\left(T^{+}\right)^{-1} G_{n}(\xi)\right]\right\}$. This may be easily proven using the unitarity of $T^{+}$and $T^{-}$. Now suppose $F=\left\{\left[F_{n}(\xi), T^{-}\left(T^{+}\right)^{-1} F_{n}(\xi)\right]\right\} \in \mathscr{S}^{E}$. It follows from (3.1) that $F=\mathscr{T}^{+} f$, where

$$
f=\frac{2}{\sqrt{ } 2}\left[A^{-1 / 2}\left(T^{+}\right)^{-1}\left\{F_{n}(\xi)\right\}, 0\right] .
$$


If $G=\left\{\left[G_{n}(\xi),-T^{-}\left(T^{+}\right)^{-1} G_{n}(\xi)\right]\right\} \in \mathscr{S}^{\circ}$, then we have $G=\mathscr{T}^{+} g$ where

$$
g=\frac{2}{\sqrt{ } 2}\left[0, \frac{-1}{i}\left(T^{+}\right)^{-1}\left\{G_{n}(\xi)\right\}\right] .
$$

Since $H^{2}=\mathscr{S}^{E} \oplus \mathscr{S}^{\circ}$, we have proven that $\mathscr{T}^{+}$is unitary.

(b) Suppose $f=\left[f_{1}, f_{2}\right] \in \mathscr{H}$. Then from (3.1), the definitions (2.3) of $\alpha_{n}^{+}(\xi)$ and $\beta_{n}^{+}(\xi)$, and similar definitions of $\alpha_{n}^{-}(\xi)$ and $\beta_{n}^{-}(\xi)$, it follows that

$$
\mathscr{T}^{+} f=\sqrt{ } 2\left\{\left[\left(\xi^{2}+v_{n}\right)^{1 / 2} \alpha_{n}^{+}(\xi),\left(\xi^{2}+v_{n}\right)^{1 / 2} \beta_{n}^{-}(\xi)\right]\right\} .
$$

Using the definition (2.7) of $U(t)$, we have

$$
\begin{aligned}
& \mathscr{T}^{+}(U(t)(f)
\end{aligned}
$$

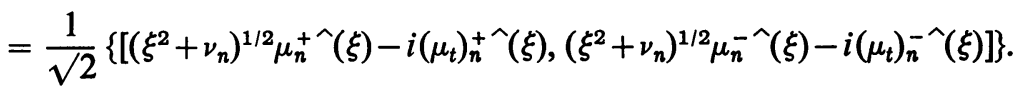

Theorem 2.1 gives

$$
\begin{aligned}
\mu_{n}^{+\uparrow}(\xi)= & \alpha_{n}^{+}(\xi) \exp \left[i\left(\xi^{2}+\nu_{n}\right)^{1 / 2} t\right]+\beta_{n}^{+}(\xi) \exp \left[-i\left(\xi^{2}+\nu_{n}\right)^{1 / 2} t\right] \\
\left(\mu_{t}\right)_{n}^{+}(\xi)= & i\left(\xi^{2}+\nu_{n}\right)^{1 / 2} \alpha_{n}^{+}(\xi) \exp \left[i\left(\xi^{2}+\nu_{n}\right)^{1 / 2} t\right] \\
& -i\left(\xi^{2}+\nu_{n}\right)^{1 / 2} \beta_{n}^{+}(\xi) \exp \left[-i\left(\xi^{2}+\nu_{n}\right)^{1 / 2} t\right] .
\end{aligned}
$$

Hence

$$
\left(\xi^{2}+\nu_{n}\right)^{1 / 2} \mu_{n}^{+\uparrow}(\xi)-i\left(\mu_{t}\right)_{n}^{+}(\xi)=2\left(\xi^{2}+\nu_{n}\right)^{1 / 2} \alpha_{n}^{+}(\xi) \exp \left[i\left(\xi^{2}+\nu_{n}\right)^{1 / 2} t\right]
$$

Similarly

$$
\left(\xi^{2}+\nu_{n}\right)^{1 / 2} \mu_{n}^{-\uparrow}(\xi)+i\left(\mu_{t}\right)_{n}^{-\uparrow}(\xi)=2\left(\xi^{2}+\nu_{n}\right)^{1 / 2} \beta_{n}^{-}(\xi) \exp \left[-i\left(\xi^{2}+\nu_{n}\right)^{1 / 2} t\right]
$$

Combining (3.5), (3.6), (3.7) and (3.8), we see that Theorem 3.1 is proven. Q.E.D.

We next obtain an expression for the inverse of $\mathscr{T}^{ \pm}$. Suppose $F=\left\{\left[F_{(1)_{n}}(\xi)\right.\right.$, $\left.\left.F_{(2)}(\xi)\right]\right\} \in H^{2}$. Using (3.1) it is easy to verify that

$$
\begin{array}{r}
\left(\mathscr{T}^{ \pm}\right)^{-1} F=\frac{1}{\sqrt{ } 2}\left[\left(T^{ \pm}\right)^{-1}\left\{\left(\xi^{2}+\nu_{n}\right)^{-1 / 2} F_{(1)_{n}}(\xi)\right\}+\left(T^{\mp}\right)^{-1}\left\{\left(\xi^{2}+\nu_{n}\right)^{-1 / 2} F_{(2)_{n}}(\xi)\right\}\right. \\
\left.\frac{1}{i}\left(T^{\mp}\right)^{-1}\left\{F_{(2)_{n}}(\xi)\right\}-\frac{1}{i}\left(T^{ \pm}\right)^{-1}\left\{F_{(1)_{n}}(\xi)\right\}\right] .
\end{array}
$$

Finally we note that a theorem analogous to Theorem 3.1 holds for the transformation $\mathscr{T}^{0}$ defined by (3.2). $\left(\mathscr{T}^{0}\right)^{-1}$ is given by

$$
\begin{aligned}
\left(\mathscr{T}^{0}\right)^{-1} F=\frac{1}{\sqrt{ } 2}\left[( T ^ { 0 } ) ^ { - 1 } \left\{( \xi ^ { 2 } + \nu _ { n } ) ^ { - 1 / 2 } \left(F_{(1)_{n}}(\xi)\right.\right.\right. & \left.\left.+F_{(2)_{n}}(\xi)\right)\right\} \\
& \left.+\frac{1}{i}\left(T^{0}\right)^{-1}\left\{F_{(2)_{n}}(\xi)-F_{(1)_{n}}(\xi)\right\}\right],
\end{aligned}
$$

where $F=\left\{\left[F_{(1)_{n}}(\xi), F_{(2)_{n}}(\xi)\right]\right\}$. 
4. The wave operators. We are interested in comparing the behavior of solutions $\mu^{0}(x, t)$ of the wave equation in $S$ with the behavior of solutions $\mu(x, t)$ of the wave equation in $\Omega$ as $t \rightarrow \pm \infty$. To this end we define the wave operators $\mathscr{W}^{ \pm}$ as follows. Suppose $\varphi=\left[\varphi_{1}, \varphi_{2}\right]$, where $\varphi_{j}(x) \in C_{0}^{\infty}(S), j=1,2$. Set $\mathscr{J}_{\varphi}=\left[\sigma \varphi_{1}, \sigma \varphi_{2}\right]$, where $\sigma(x)$ satisfies the following conditions:

(i) $\sigma(x) \in C^{\infty}(\Omega)$,

(ii) $\sigma(x) \equiv 0$ for $\left|x_{N}\right| \leqq h$, and

(iii) $\sigma(x) \equiv 1$ for $h^{\prime} \leqq\left|x_{N}\right|$, where $h<h^{\prime}$.

Recall that for $h \leqq\left|x_{N}\right|$, we have $\Omega=S$. Extend $\mathscr{J}$ by continuity to all of $\mathscr{H}_{0}$.

Suppose $f=\left[f_{1}, f_{2}\right] \in \mathscr{H}_{0}$. Set $\mathscr{W}(t) f=\mathscr{U}(-t) \mathscr{J} \mathscr{U}^{0}(t) f$. We shall prove in the next theorem that $\mathscr{W}^{ \pm} f=\lim _{t \rightarrow \pm \infty} \mathscr{W}(t) f$ exists in $\mathscr{H}_{0}$ and may be given by $\left(\mathscr{T}^{ \pm}\right)^{-1} \mathscr{T}^{0}$. From this it will follow that $\mathscr{W}^{ \pm}$are a pair of unitary mappings from $\mathscr{H}_{0}$ onto $\mathscr{H}_{\text {. }}$

TheOREM 4.1. Suppose $f=\left[f_{1}, f_{2}\right] \in \mathscr{H}_{0}$. Then

$$
\mathscr{W}^{ \pm} f=\lim _{t \rightarrow \pm \infty} \mathscr{W}(t) f=\left(\mathscr{T}^{ \pm}\right)^{-1} \mathscr{T}^{0} f \quad \text { in } \mathscr{H} .
$$

Proof. We shall carry through the proof for the case $t \rightarrow+\infty$. Our aim is to prove that

$$
\begin{aligned}
\lim _{t \rightarrow \infty} \mathscr{T}^{+} \mathscr{W}(t) f & =\mathscr{T}_{0} f \\
& =\frac{1}{\sqrt{ } 2}\left\{\left[\left(\xi^{2}+\nu_{n}\right)^{1 / 2} \hat{f}_{(1)_{n}}^{0}(\xi)-i \hat{f}_{(2)_{n}}^{0}(\xi),\left(\xi^{2}+v_{n}\right)^{1 / 2} \hat{f}_{(1)_{n}}^{0}(\xi)+i \hat{f}_{(2)_{n}}^{0}(\xi)\right]\right\}
\end{aligned}
$$

in $H^{2}$. We shall establish (4.1) for the following dense subset $\mathscr{D}$ of $\mathscr{H}_{0}$. $\mathscr{D}$ consists of all those elements $\varphi=\left[\varphi_{1}, \varphi_{2}\right]$ in $\mathscr{H}_{0}$ satisfying the conditions

(i) $\hat{\varphi}_{(j)_{n}}^{0}(\xi) \equiv 0$ for $n \geqq M$,

(ii) each $\hat{\varphi}_{(j)_{n}}^{0}(\xi) \in C_{0}^{\infty}\left(R^{1}\right)$, and

(iii) each $\hat{\varphi}_{(j)_{n}}^{0}(\xi)$ vanishes in a neighborhood of each of the points $\pm\left(\nu_{\kappa}-v_{n}\right)^{1 / 2}$, $\kappa=1,2, \ldots$, where $j=1,2$ and $M$ is a positive integer.

Employing (3.1) and Theorem 3.1, we obtain

$$
\begin{aligned}
\mathscr{T}^{+\mathscr{W}(t) \varphi}= & \frac{1}{\sqrt{ } 2}\left\{\left[\Phi_{(1)_{n}}(\xi ; t), \Phi_{(2)_{n}}(\xi ; t)\right]\right\} \\
= & \frac{1}{\sqrt{ } 2}\left\{\left[\begin{array}{c}
\exp \left[-i\left(\xi^{2}+\nu_{n}\right)^{1 / 2} t\right] \\
\times\left[\left(\xi^{2}+\nu_{n}\right)^{1 / 2}\left(\sigma \mu^{0}\right)_{n}^{+\wedge}(\xi)-i\left(\sigma \mu_{t}^{0}\right)_{n}^{+\wedge}(\xi)\right] \\
\exp \left[i\left(\xi^{2}+\nu_{n}\right)^{1 / 2} t\right] \\
\times\left[\left(\xi^{2}+\nu_{n}\right)^{1 / 2}\left(\sigma \mu^{0}\right)_{n}^{-\wedge}(\xi)+i\left(\sigma \mu_{t}^{0}\right)_{n}^{-\wedge}(\xi)\right]
\end{array}\right]\right\}
\end{aligned}
$$

Consider $\Phi_{(1)_{n}}(\xi ; t)$. First we make some definitions. For each function $f(x)$ in $L_{2}(S)\left[L_{2}(\Omega)\right]$, set $U_{0}(t) f=\exp \left(i t A_{0}^{1 / 2}\right) f\left[U(t) f=\exp \left(i t A^{1 / 2}\right) f\right]$. Suppose $\psi(x) \in$ $\dot{C}_{0}^{\infty}(S)$. Set $J \psi(x)=\sigma(x) \psi(x)$ and extend $J$ by continuity to all of $L_{2}(S)$. Finally, set $W(t) f=U(-t) J U_{0}(t) f$ for each $f$ in $L_{2}(S)$. 
Using the properties of the spectral representations $T_{0}, T^{+}$, and $T^{-}$as well as the inversion formula (3.10), we have

$$
\begin{aligned}
& \Phi_{(1)_{n}}(\xi ; t)=\frac{1}{2}\left(\xi^{2}+\nu_{n}\right)^{1 / 2}\left[\left(W(t) \varphi_{(1)_{n}}\right)^{+\uparrow}(\xi)-i\left(W(t) A_{0}^{-1 / 2} \varphi_{(2)_{n}}\right)^{+\wedge}(\xi)\right. \\
& \left.+\left(U(-t) J U_{0}(-t) \varphi_{(1)_{n}}\right)^{+\wedge}(\xi)+i\left(U(-t) J U_{0}(-t) A_{0}^{-1 / 2} \varphi_{(2)_{n}}\right)^{+\wedge}(\xi)\right] \\
& -\frac{1}{2}\left[-\left(W(t) A_{0}^{1 / 2} \varphi_{(1)_{n}}\right)^{+\uparrow}(\xi)+i\left(W(t) \varphi_{(2)_{n}}\right)^{+\uparrow}(\xi)\right. \\
& \left.+\left(U(-t) J U_{0}(-t) A_{0}^{1 / 2} \varphi_{(1)_{n}}\right)^{+\uparrow}(\xi)+i\left(U(-t) J U_{0}(-t) \varphi_{(2)_{n}}\right)^{+\uparrow}(\xi)\right] .
\end{aligned}
$$

Rearranging the terms on the right hand side of the preceding equation and making repeated use of the triangle inequality, we obtain the following inequality

$$
\begin{aligned}
& \left\|\Phi_{(1)_{n}}(\xi ; t)-\left[\left(\xi^{2}+\nu_{n}\right)^{1 / 2} \hat{\varphi}_{(1)_{n}}^{0}(\xi)-i \hat{\varphi}_{(2)_{n}}^{0}(\xi)\right]\right\|_{H} \\
& \leqq \frac{1}{2}\left\|\left(\xi^{2}+v_{n}\right)^{1 / 2}\left[\left(W(t) \varphi_{(1)_{n}}\right)^{+\uparrow}(\xi)-\hat{\varphi}_{(1)_{n}}^{0}(\xi)\right]\right\|_{H} \\
& +\frac{1}{2}\left\|\left(W(t) A_{0}^{1 / 2} \varphi_{(1)_{n}}\right)^{+\wedge}(\xi)-\left(A_{0}^{1 / 2} \varphi_{(1)_{n}}\right)^{0 \wedge}(\xi)\right\|_{H} \\
& +\frac{1}{2}\left\|i \hat{\varphi}_{(2)_{n}}^{0}(\xi)-i(W(t) \varphi)_{(2)_{n}}^{+\hat{n}}(\xi)\right\|_{H} \\
& +\frac{1}{2}\left\|i \hat{\varphi}_{(2) n}^{0}(\xi)-i\left(A^{1 / 2} W(t) A_{0}^{-1 / 2} \varphi_{(2)}\right)_{n}^{+\uparrow}(\xi)\right\|_{H} \\
& +\frac{1}{2}\left\|\left(A^{1 / 2} U(-t) J U_{0}(-t) \varphi_{(1)}\right)_{n}^{+\wedge}(\xi)-\left(U(-t) J A_{0}^{1 / 2} U_{0}(-t) \varphi_{(1)}\right)_{n}^{+^{\wedge}}(\xi)\right\|_{H} \\
& +\frac{1}{2} \| i\left(A^{1 / 2} U(-t) J A_{0}^{-1 / 2} U_{0}(-t) \varphi_{(2)}\right)_{n}^{+\uparrow}(\xi) \\
& -i\left(U(-t) J U_{0}(-t) \varphi_{(2)}\right)_{n}^{+}(\xi) \|_{H} \\
& =\frac{1}{2}\left[\left\|I_{1}(\cdot, t)\right\|_{H}+\left\|I_{2}(\cdot, t)\right\|_{H}+\left\|I_{3}(\cdot, t)\right\|_{H}+\left\|I_{4}(\cdot, t)\right\|_{H}\right. \\
& \left.+\left\|I_{5}(\cdot, t)\right\|_{H}+\left\|I_{6}(\cdot, t)\right\|_{H}\right] .
\end{aligned}
$$

In order to prove that the right hand side of (4.4) $\rightarrow 0$ as $t \rightarrow \infty$, we establish the following lemma.

Lemma 4.1. Suppose $\psi(x) \in L_{2}(S)$ and $T_{0} \psi$ satisfies conditions (i)-(iii). Also, suppose the bounded interval $[a, b]$ does not contain any of the points $\left\{\nu_{\kappa}\right\}, \kappa=1,2, \ldots$ Then

$$
\lim _{t \rightarrow \pm \infty}(W(t) \psi)_{n}^{ \pm}{ }^{\wedge}(\xi)=\hat{\psi}_{n}^{0}(\xi)
$$

uniformly in $\xi$ and $n$ provided $\xi^{2}+\nu_{n} \in[a, b]$.

Proof. Again we consider $t \rightarrow+\infty$. Using the spectral representations $T^{0}$ and $T^{+}$, we have

$$
\begin{aligned}
(W(t) \psi)_{n}^{+}(\xi)= & \operatorname{lim.m} . \int_{\Omega_{M}} \bar{w}_{n}^{+}(x ; \xi) \sigma(x) \\
& \cdot\left(\sum_{j=1}^{\infty} \int_{-\infty}^{\infty} w_{j}^{0}(x ; \zeta) \exp \left\{i t\left[\left(\zeta^{2}+\nu_{j}\right)^{1 / 2}-\left(\xi^{2}+\nu_{n}\right)^{1 / 2}\right]\right\} \hat{\psi}_{j}^{0}(\zeta) d \zeta\right) d x
\end{aligned}
$$


where $\Omega_{M}$ consists of those points in $\Omega$ for which $\left|x_{N}\right| \leqq M$. It follows from I that

$$
\max _{x \in \Omega}\left|w_{n}^{+}(x ; \xi)\right| \leqq C
$$

uniformly for $\xi^{2}+v_{n} \in[a, b]$. Furthermore, integration by parts yields

$$
\begin{aligned}
\left|x_{N}^{J} U^{0}(t) \psi(x)\right| & =\left|x_{N}^{J} \sum_{j=1}^{\infty} \int_{-\infty}^{\infty} w_{j}^{0}(x ; \zeta) \exp \left[i t\left(\zeta^{2}+v_{j}\right)^{1 / 2}\right] \hat{\psi}_{j}^{0}(\zeta) d \zeta\right| \\
& =\left|\sum_{j=1}^{\infty} \int_{-\infty}^{\infty} \frac{\partial^{J}}{\partial(i \zeta)^{J}}\left(\exp \left[i \zeta x_{N}\right]\right) \eta_{j}(\tilde{x}) \exp \left[i t\left(\zeta^{2}+\nu_{j}\right)^{1 / 2}\right] \hat{\psi}_{j}^{0}(\zeta) d \zeta\right| \\
& =\left|\sum_{j=1}^{\infty} \int_{-\infty}^{\infty} \exp \left[i \zeta x_{N}\right] \frac{\partial^{J}}{\partial(i \zeta)^{J}}\left(\eta_{j}(\tilde{x}) \exp \left[i t\left(\zeta^{2}+\nu_{j}\right)^{1 / 2}\right] \hat{\psi}_{j}^{0}(\zeta)\right) d \zeta\right| \\
& \leqq C \text { for each } J=1,2, \ldots
\end{aligned}
$$

Hence

$$
U_{0}(t) \psi(x)=O\left(\left|x_{N}\right|^{-J}\right)
$$

(Similarly it follows that $D^{\kappa} U_{0}(t) \psi(x)=O\left(\left|x_{N}\right|^{-J}\right.$ ) for $\left.\kappa=1,2, \ldots\right)$ (4.6) combined with (4.7) shows that the right hand side of (4.5) converges in the ordinary sense, i.e.

$$
\begin{aligned}
(W(t) \psi)_{n}^{+^{\curlywedge}}(\xi)= & \int_{\Omega} \bar{w}_{n}^{+}(x ; \xi) \sigma(x) \sum_{j=1}^{\infty} \int_{-\infty}^{\infty} w_{j}^{0}(x ; \zeta) \\
& \cdot \exp \left\{i t\left[\left(\zeta^{2}+\nu_{j}\right)^{1 / 2}-\left(\xi^{2}+\nu_{n}\right)^{1 / 2}\right]\right\} \hat{\psi}_{j}^{0}(\zeta) d \zeta d x
\end{aligned}
$$

Differentiating both sides of (4.8) with respect to $t$, we obtain

$$
\begin{aligned}
\frac{d}{d t}(W(t) \psi)_{n}^{+\wedge}(\xi)= & i \int_{\Omega} \bar{w}_{n}^{+}(x ; \xi) \sigma(x) d x \\
& \sum_{j=1}^{\infty} \int_{-\infty}^{\infty}\left[\left(\zeta^{2}+\nu_{j}\right)-\left(\xi^{2}+\nu_{n}\right)\right] L_{j, n}(\zeta, \xi) \\
& \cdot \exp \left\{i t\left[\left(\zeta^{2}+\nu_{j}\right)^{1 / 2}-\left(\xi^{2}+\nu_{n}\right)^{1 / 2}\right]\right\} w_{j}^{0}(x, \zeta) \hat{\psi}_{j}^{0}(\zeta) d \zeta,
\end{aligned}
$$

where

$$
L_{j, n}(\zeta, \xi)=\left[\left(\zeta^{2}+\nu_{j}\right)^{1 / 2}-\left(\xi^{2}+\nu_{n}\right)^{1 / 2}\right] /\left[\left(\zeta^{2}+v_{j}\right)-\left(\xi^{2}+v_{n}\right)\right] .
$$

$L_{j, n}(\zeta, \xi)$ is a $C^{\infty}$ function of $\left(\zeta^{2}+\nu_{j}\right)^{1 / 2}$ for $\zeta$ in the support of $\hat{\psi}_{j}^{0}(\zeta)$.

Set

$$
V_{n}(x ; \xi ; t ; \varepsilon)=i \sum_{j=1}^{\infty} \int_{-\infty}^{\infty} w_{j}^{0}(x ; \zeta) L_{j, n}(\zeta, \xi) \hat{\psi}_{j}^{0}(\zeta)
$$

$$
\cdot \exp \left\{i t\left[\left(\zeta^{2}+\nu_{j}\right)^{1 / 2}-\left(\xi^{2}+\nu_{n}\right)^{1 / 2}+i \varepsilon L_{j, n}(\zeta, \xi)\right]\right\} d \zeta
$$


Again, integration by parts shows that

$$
D_{x}^{\alpha} V_{n}(x ; \xi ; t ; \varepsilon)=O\left(\left(1+\left|x_{N}\right|\right)^{-M}\right) \text { for } M=1,2, \ldots,
$$

and all multiple indices $\alpha$.

Using (4.9), (4.11), integration by parts, the equation

$$
\Delta w_{j}^{0}(x ; \zeta)=-\left(\zeta^{2}+v_{j}\right) w_{j}^{0}(x ; \zeta)
$$

and the boundary condition $w_{j}^{0}(x ; \zeta)=0$ on $\dot{S}$, we have

$$
\frac{d}{d t}(W(t) \psi)_{n}^{+\wedge}(\xi)=-\int_{\Omega} V_{n}(x ; \xi ; t ; 0)\left(\Delta+\left(\xi^{2}+\nu_{n}\right)\right)\left[\sigma(x) w_{n}^{+}(x ; \xi)\right] d x
$$

Note that $\left(\Delta+\xi^{2}+v_{n}\right)\left[\sigma(x) w_{n}^{+}(x ; \xi)\right]$ has compact support in $x$ since $\sigma(x) \equiv 1$ for $\left|x_{N}\right| \geqq h^{\prime}$. Equation (4.12) and the definition of $W(t)$ imply

$$
\begin{aligned}
(W(T) \psi)_{n}^{+\wedge}(\xi)= & (\sigma \psi)_{n}^{+\uparrow}(\xi)-\int_{0}^{T} d t \int_{\Omega} V_{n}(x ; \xi ; t ; 0) \\
& \cdot\left(\Delta+\left(\xi^{2}+\nu_{n}\right)\right)\left[\sigma(x) w_{n}^{+}(x ; \xi)\right] d x .
\end{aligned}
$$

To show that the limit on the right hand side of (4.13) exists as $T \rightarrow \infty$, we prove that

$$
V_{n}(x ; \xi ; t ; \varepsilon)=O\left((1+|t|)^{-2}\right)
$$

uniformly for $x$ in compact subsets of $S, \xi^{2}+\nu_{n}$ in $[a, b]$, all $t$, and $0 \leqq \varepsilon$ sign $t \leqq 1$. Set

$$
M_{j, n}(\zeta, \xi, \varepsilon)=\frac{\partial\left[\left(\zeta^{2}+\nu_{j}\right)^{1 / 2}-\left(\xi^{2}+\nu_{n}\right)^{1 / 2}+i \varepsilon L_{j, n}(\zeta, \xi)\right]}{\partial\left(\left(\zeta^{2}+\nu_{j}\right)^{1 / 2}\right)}
$$

Multiplying both sides of (4.11) by $t^{2}$, we have

$$
\begin{aligned}
t^{2} V_{n}(x ; \xi ; t ; \varepsilon)= & -i \sum_{j=1}^{\infty} \int_{-\infty}^{\infty} w_{j}^{0}(x ; \zeta) \hat{\psi}_{j}^{0}(\zeta) L_{j, n}(\zeta, \xi) M_{j, n}^{-1}(\zeta, \xi, \varepsilon) \frac{\partial}{\partial\left(\left(\zeta^{2}+\nu_{j}\right)^{1 / 2}\right)} \\
& \cdot\left(M_{j, n}^{-1}(\zeta, \xi, \varepsilon) \frac{\partial}{\partial\left(\left(\zeta^{2}+\nu_{j}\right)^{1 / 2}\right)}\right. \\
& \left.\cdot \exp \left\{i t\left[\left(\zeta^{2}+v_{j}\right)^{1 / 2}-\left(\xi^{2}+v_{n}\right)^{1 / 2}+i \varepsilon L_{j, n}(\zeta, \xi)\right]\right\}\right) d \zeta .
\end{aligned}
$$

It is clear from the definition of $M_{j, n}(\zeta, \xi, \varepsilon)$ that $\left|M_{j, n}(\zeta, \xi, \varepsilon)\right| \geqq 1$. Thus $\left|M_{j, n}^{-1}(\zeta, \xi)\right| \leqq C$. Integrating by parts twice in (4.15) and using the fact that $L_{j, n}(\zeta, \xi) \geqq 0$, we conclude that (4.14) holds and hence the limit in (4.13) exists. We next evaluate this limit.

It is evident from the definition (4.11) that

$$
V_{n}(x ; \xi ; t ; \varepsilon) \rightarrow V_{n}(x ; \xi ; t ; 0) \text { as } \varepsilon \rightarrow 0
$$


uniformly for $\left|x_{N}\right|$ bounded, $\xi^{2}+v_{n}$ in $[a, b]$ and bounded $t$. Thus

$$
\begin{aligned}
\lim _{\varepsilon \downarrow 0} \int_{0}^{T} V_{n}(x ; \xi ; t ; \varepsilon)\left(\Delta+\left(\xi^{2}+\nu_{n}\right)\right)\left[\sigma(x) w_{n}^{+}(x ; \xi)\right] d x \\
=\int_{0}^{T} V_{n}(x ; \xi ; t ; 0)\left(\Delta+\left(\xi^{2}+\nu_{n}\right)\right)\left[\sigma(x) w_{n}^{+}(x ; \xi)\right] d x .
\end{aligned}
$$

Furthermore, it follows from (4.14) that

$$
\lim _{T \rightarrow \infty} \int_{0}^{T} d t \int_{\Omega} V_{n}(x ; \xi ; t ; \varepsilon)\left(\Delta+\xi^{2}+\nu_{n}\right)\left[\sigma(x) w_{n}^{+}(x ; \xi)\right] d x
$$

exists uniformly for $0 \leqq \varepsilon \leqq 1$. Therefore, by the double limit theorem

$$
\begin{aligned}
-\lim _{T \rightarrow \infty} \lim _{\varepsilon \downarrow 0} \int_{0}^{T} d t \int_{\Omega} V_{n}(x ; \xi ; t ; \varepsilon)\left(\Delta+\xi^{2}+\nu_{n}\right)\left[\sigma(x) \bar{w}_{n}^{+}(x ; \xi)\right] d x \\
\quad=-\lim _{\varepsilon \downarrow 0} \lim _{T \rightarrow \infty} \int_{0}^{T} d t \int_{\Omega} V_{n}(x ; \xi ; t ; \varepsilon)\left(\Delta+\xi^{2}+\nu_{n}\right)\left[\sigma(x) \bar{w}_{n}^{+}(x ; \xi)\right] d x
\end{aligned}
$$

According to equation (4.13), the left hand side of (4.16) is equal to

$$
\lim _{T \rightarrow \infty}(W(T) \psi)_{n}^{+\wedge}(\xi)-(\sigma \psi)_{n}^{+\wedge}(\xi) .
$$

We shall show that the right hand side of (4.16) has the value

$$
\int_{S} K_{n}^{+}(x ; \xi) \psi(x) d x, \quad \text { where } K_{n}^{+}(x ; \xi)=\bar{w}_{n}^{0}(x ; \xi)-\sigma(x) \bar{w}_{n}^{+}(x ; \xi) .
$$

(We have extended $\sigma(x) \bar{w}_{n}^{+}(x ; \xi)$ to $S-\Omega$ by defining it to be zero there.) Once we establish that the limit in (4.16) is equal to (4.18), the lemma will be proven.

Since $\left(\Delta+\xi^{2}+v_{n}\right) w_{n}^{0}(x ; \xi)=0$, we may replace $-\sigma(x) \bar{w}_{n}^{+}(x ; \xi)$ in (4.16) by $K_{n}^{+}(x ; \xi)$. Interchanging the order of the integrals, we see that the right hand side of (4.16) is equal to

$$
\lim _{\varepsilon \downarrow 0} \int_{S}\left(\Delta+\xi^{2}+\nu_{n}\right) K_{n}^{+}(x ; \xi) \int_{0}^{\infty} V_{n}(x ; \xi ; t ; \varepsilon) d t d x .
$$

Employing the spectral representation $T^{0}$, we have

$$
\begin{aligned}
\int_{0}^{\infty} V_{n}(x ; \xi ; t ; \varepsilon) d t & =-\sum_{j=1}^{\infty} \int_{-\infty}^{\infty} w_{j}^{0}(x ; \zeta) \hat{\psi}_{j}^{0}(\zeta) \\
& \cdot \frac{L_{j, n}(\zeta, \xi) d \zeta}{\left[\left(\zeta^{2}+v_{j}\right)^{1 / 2}-\left(\xi^{2}+v_{n}\right)^{1 / 2}+i \varepsilon L_{j, n}(\zeta, \xi)\right]} \\
& =-\sum_{j=1}^{\infty} \int_{-\infty}^{\infty} w_{j}^{0}(x ; \zeta)\left[\left(\zeta^{2}+v_{j}\right)-\left(\xi^{2}+v_{n}\right)+i \varepsilon\right]^{-1} \hat{\psi}_{j}^{0}(\zeta) d \zeta \\
& =-\left[A_{0}-\left(\xi^{2}+v_{n}-i \varepsilon\right)\right]^{-1} \psi(x) \rightarrow z_{n}(x ; \xi)
\end{aligned}
$$

uniformly for $x$ in the support of $\left[\Delta+\xi^{2}+v_{n}\right] K_{n}^{+}(x ; \xi)$, where the function $z_{n}(x ; \xi)$ satisfies the boundary value problem

$$
\left(\Delta+\xi^{2}+\nu_{n}\right) z_{n}(x ; \xi)=\psi(x) \text { in } S,
$$

$z_{n}(x ; \xi)=0$, on $\dot{S}$, as well as the "incoming" radiation conditions at infinity. Therefore $\int_{0}^{\infty} V_{n}(x ; \xi ; t ; \varepsilon) d t \rightarrow z_{n}(x ; \xi)$. 
Since the first integrand in (4.19) has compact support we may take the limit under the first integral. Thus (4.19) may be expressed as

$$
\int_{S} z_{n}(x ; \xi)\left(\Delta+\xi^{2}+\nu_{n}\right) K_{n}^{+}(x ; \xi) d x .
$$

Since for $\left|x_{N}\right|$ large, $K_{n}^{+}(x ; \xi)=-\bar{v}_{n}^{+}(x ; \xi)$ and $z_{n}(x ; \xi)$ both satisfy the incoming radiation conditions, we may integrate by parts and use (4.20) to conclude that (4.21) is equal to (4.18). The lemma is thus proven. Q.E.D.

We next want to show that each term on the right hand side of (4.4) $\rightarrow 0$ as $t \rightarrow \infty$. Consider $I_{2}$. Suppose $t_{j} \rightarrow \infty$ as $j \rightarrow \infty$. The sequence of elements $\left\{T^{+}\left(W\left(t_{j}\right) A_{0}^{1 / 2} \varphi_{(1)}\right)\right\}$ satisfies the condition

$$
T^{+}\left(W\left(t_{j}\right) A_{0}^{1 / 2} \varphi_{(1)}\right) \rightarrow T^{0} A_{0}^{1 / 2} \varphi_{(1)}
$$

in $H$ as $t_{j} \rightarrow \infty$ ( $\rightarrow$ denotes weak convergence in the Hilbert space). To prove this we note that since $\varphi_{(1)}(x)$ satisfies the hypothesis of Lemma $4.1, A_{0}^{1 / 2} \varphi_{(1)}(x)$ does also. Hence it follows from Lemma 4.1 that

$$
\left(\left(W\left(t_{j}\right) A_{0}^{1 / 2} \varphi_{(1)}\right)_{n}^{+\wedge}(\xi), \chi_{[a, b]}\left(\xi^{2}+\nu_{n}\right)\right)_{H} \rightarrow\left(\left(A_{0}^{1 / 2} \varphi_{(1)}\right)_{n}^{0 \wedge}(\xi), \chi_{[a, b]}\left(\xi^{2}+\nu_{n}\right)\right)_{H}
$$

as $j \rightarrow \infty$, where $[a, b]$ is an arbitrary bounded interval not containing any of the points $\left\{\nu_{i}\right\}$ and $\chi_{[a, b]}$ denotes the characteristic function of the interval $[a, b]$. Since the elements $\left\{\chi_{[a, b]}\left(\xi^{2}+\nu_{n}\right)\right\}$ form a dense subset of $H$, (4.22) follows from (4.23).

We show that

$$
\left\|T^{+}\left(W\left(t_{j}\right) A_{0}^{1 / 2} \varphi_{(1)}\right)\right\|_{H} \rightarrow\left\|T^{0} A_{0}^{1 / 2} \varphi_{(1)}\right\|_{H} \quad \text { as } j \rightarrow \infty .
$$

Using the isometric property of the transformations $T_{0}, T^{+}, U_{0}(t)$, and $U(t)$, then applying the divergence theorem, we have

$$
\begin{aligned}
& \left|\left\|T^{+}\left(W\left(t_{j}\right) A_{0}^{1 / 2} \varphi_{(1)}\right)\right\|_{H}^{2}-\left\|T_{0} A_{0}^{1 / 2} \varphi\right\|_{H}^{2}\right| \\
& =|| J U_{0}\left(t_{j}\right) A_{0}^{1 / 2} \varphi_{(1)}\left\|_{L_{2}(\Omega)}^{2}-\right\| U_{0}\left(t_{j}\right) A_{0}^{1 / 2} \varphi_{(1)} \|_{L_{2}(S)}^{2} \mid \\
& =\mid \int_{S} \sigma(x) A_{0}^{1 / 2} U_{0}\left(t_{j}\right) \varphi_{(1)}(x) \bar{\sigma}(x)\left(A_{0}^{1 / 2} U_{0}\left(t_{j}\right) \varphi_{(1)}(x)\right)^{*} d x \\
& \quad \quad-\int_{S} A_{0}^{1 / 2} U_{0}\left(t_{j}\right) \varphi_{(1)}(x)\left(A_{0}^{1 / 2} U_{0}\left(t_{j}\right) \varphi_{(1)}(x)\right)^{*} d x \mid \\
& =\mid \int_{S} A_{0}^{1 / 2} U_{0}\left(t_{j}\right) \varphi_{(1)}(x)\left(A_{0}^{1 / 2} U_{0}\left(t_{j}\right) \varphi_{(1)}(x)\right)^{*} d x \\
& \quad+\int_{S}\left(\sigma(x)^{2}-1\right) A_{0}^{1 / 2} U_{0}\left(t_{j}\right) \varphi_{(1)}(x)\left(A_{0}^{1 / 2} U_{0}\left(t_{j}\right) \varphi_{(1)}(x)\right)^{*} d x \\
& =\left.\left|\int_{S}\left(\sigma(x)^{2}-1\right)\right| A_{0}^{1 / 2} U_{0}\left(t_{j}\right) \varphi_{(1)}(x)\right|^{2} d x \mid \\
& \leqq C \int_{B}\left|A_{0}^{1 / 2} U_{0}\left(t_{j}\right) \varphi_{(1)}(x)\left(A_{0}^{1 / 2} U_{0}\left(t_{j}\right) \varphi_{(1)}(x)\right)^{*} d x\right|
\end{aligned}
$$


where $B$ is the support of $\sigma(x)^{2}-1$. Note that $B$ is a compact subset of $S$. Using the spectral representation $T_{0}$ and integration by parts as well as properties (i)-(iii) of $T^{0} \varphi_{(1)}$, it follows easily that

$$
\max _{x \in B}\left|A_{0}^{1 / 2} U_{0}(t) \varphi_{(1)}(x)\right|=O\left(|t|^{-1}\right) \text { for large }|t| .
$$

This completes the proof of (4.24).

We now apply the following well-known theorem. Given a sequence of elements $h_{j}$ and an element $h$ satisfying the following conditions in a Hilbert space:

(a) $h_{j} \rightarrow h$ as $j \rightarrow \infty$, and

(b) $\left\|h_{j}\right\| \rightarrow\|h\|$ as $j \rightarrow \infty$.

Then $\left\|h_{j}-h\right\| \rightarrow 0$ as $j \rightarrow \infty$.

In our case (4.22) and (4.24) show that the sequence $T\left(W\left(t_{j}\right) A_{0}^{1 / 2} \varphi_{(1)}\right)$ and the element $T_{0} A_{0}^{1 / 2} \varphi_{(1)}$ satisfy conditions (a) and (b) in $H$. Hence we conclude that $\left\|I_{2}(\cdot, t)\right\|_{H}$ $\rightarrow 0$ as $t \rightarrow \infty$. Similar reasoning shows that $\left\|I_{1}(\cdot, t)\right\|_{H},\left\|I_{3}(\cdot, t)\right\|_{H}$ and $\left\|I_{4}(\cdot, t)\right\|_{H}$ $\rightarrow 0$ as $t \rightarrow \infty$.

Finally, consider $\left\|I_{5}\right\|$ and $\left\|I_{6}\right\|$. Since $T^{+}$and $U(t)$ are isometric, we have

$$
\left\|I_{5}(\cdot, t)\right\|_{H}=\left\|A^{1 / 2} U\left(-t^{\prime}\right) J U_{0}\left(t^{\prime}\right) \varphi_{(1)}-U\left(-t^{\prime}\right) J A_{0}^{1 / 2} U_{0}\left(t^{\prime}\right) \varphi_{(1)}\right\|_{L_{2}(\Omega)}
$$

and

$$
\left\|I_{6}(\cdot, t)\right\|_{H}=\left\|i A^{1 / 2} U\left(-t^{\prime}\right) J A_{0}^{-1 / 2} U_{0}\left(t^{\prime}\right) \varphi_{(2)}-i U\left(-t^{\prime}\right) J U_{0}\left(t^{\prime}\right) \varphi_{(2)}\right\|_{L_{2}(\Omega)},
$$

where $t^{\prime}=-t$.

$$
\begin{aligned}
\left\|I_{5}(\cdot, t)\right\|_{H} \leqq & \left\|A^{1 / 2} W\left(t^{\prime}\right) \varphi_{(1)}-\left(T^{-}\right)^{-1} T_{0} A_{0}^{1 / 2} \varphi_{(1)}\right\|_{L_{2}(\Omega)} \\
& +\left\|\left(T^{-}\right)^{-1} T_{0} A_{0}^{1 / 2} \varphi_{(1)}-W\left(t^{\prime}\right) A_{0}^{1 / 2} \varphi_{(1)}\right\|_{L_{2}(\Omega)} .
\end{aligned}
$$

Since $t^{\prime} \rightarrow-\infty$ as $t \rightarrow+\infty$, we may apply Lemma 4.1 and the same arguments as before to show that $\left\|I_{5}(\cdot, t)\right\|_{H} \rightarrow 0$ as $t \rightarrow \infty$. Similarly $\left\|I_{6}(\cdot, t)\right\|_{H} \rightarrow 0$ as $t \rightarrow \infty$.

We have thus proven that

$$
\lim _{t \rightarrow \infty}\left\{\Phi_{(1)_{n}}(\xi, t)\right\}=\left\{\left(\xi^{2}+v_{n}\right)^{1 / 2} \hat{\varphi}_{(1)_{n}}^{0}(\xi)-i \hat{\varphi}_{(2)_{n}}^{0}(\xi)\right\} \text { in } H .
$$

It follows in the same way that

$$
\lim _{t \rightarrow \infty}\left\{\Phi_{(2)_{n}}(\xi, t)\right\}=\left\{\left(\xi^{2}+v_{n}\right)^{1 / 2} \hat{\varphi}_{(1)_{n}}^{0}(\xi)+i \hat{\varphi}_{(2)_{n}}^{0}(\xi)\right\} \text { in } H .
$$

Since the elements $\varphi=\left[\varphi_{1}, \varphi_{2}\right]$ are dense in $\mathscr{H}_{0}$, we have proven (4.2). Similarly it follows using the definition (3.1) of $\mathscr{T}^{-}$that $\lim _{t \rightarrow-\infty} \mathscr{T}^{-\mathscr{W}}(t) f=\mathscr{T}_{0} f$ in $H^{2}$. Thus the theorem is proven. Q.E.D.

We may remove the restriction that the operator $A$ has no point eigenvalues. All of the results of \$\$2-4 remain true with eigenvalues present. The unitary groups $\mathscr{U}(t)$ and the spectral representations $\mathscr{T}^{ \pm}$are constructed in the same way. In order to prove Theorem 4.1 for this case, we need the following lemma. 
Lemma 4.2. Suppose $\psi(x)$ satisfies the hypothesis of Lemma 4.1. Suppose also that $\lambda$ is an eigenvalue of $A$ of multiplicity $m$ and $w^{(1)}(x), \ldots, w^{(m)}(x)$ are the associated orthonormal eigenfunctions. Then

$$
\lim _{t \rightarrow \pm \infty}\left(W(t) \psi, w^{(j)}\right)_{(\Omega)}=0, \quad 1 \leqq j \leqq m .
$$

Proof. It follows from I that $m<\infty$. Let $P_{j}$ denote the space spanned by the eigenfunction $w^{(j)}(x)$. Using the unitarity of $U(t)$ and the definition of $P_{j}$, we have

$$
\begin{aligned}
\left|t\left(W(t) \psi, w^{(j)}\right)_{(\Omega)}\right|^{2} & =\left|t\left(P_{j} W(t) \psi, w^{(j)}\right)_{(\Omega)}\right|^{2} \\
& \leqq\left\|t P_{j} W(t) \psi\right\|_{L_{2}(\Omega)}^{2}\left\|w^{(j)}\right\|_{L_{2}(\Omega)}^{2} \\
& =\left\|t U(-t) P_{j} J U_{0}(t) \psi\right\|_{L_{2}(\Omega)}^{2} \\
& =\left\|t P_{j} J U_{0}(t) \psi\right\|_{L_{2}(\Omega)}^{2} .
\end{aligned}
$$

Now employ the spectral representation $T^{0}$ and integrate by parts. Thus

$$
\begin{aligned}
\left|t U_{0}(t) \psi(x)\right| & =\left|\sum_{n=1}^{\infty} \int_{-\infty}^{\infty} t\left[\exp \left(i\left(\xi^{2}+v_{n}\right)^{1 / 2} t\right)\right] \hat{\psi}_{n}^{0}(\xi) w_{n}^{0}(x ; \xi) d \xi\right| \\
& =\left|-i \sum_{n=1}^{\infty} \int_{-\infty}^{\infty} \frac{\partial}{\partial\left(\left(\xi^{2}+v_{n}\right)^{1 / 2}\right)}\left[\exp \left(i\left(\xi^{2}+v_{n}\right)^{1 / 2} t\right)\right] w_{n}^{0}(x ; \xi) \hat{\psi}_{n}^{0}(\xi) d \xi\right| \\
& =\left|i \sum_{n=1}^{\infty} \int_{-\infty}^{\infty} \exp \left(i\left(\xi^{2}+v_{n}\right)^{1 / 2} t\right) \frac{\partial}{\partial\left(\left(\xi^{2}+v_{n}\right)^{1 / 2}\right)}\left(\hat{\psi}_{n}^{0}(\xi) w_{n}^{0}(x ; \xi)\right) d \xi\right| \\
& \leqq C\left|x_{N}\right| .
\end{aligned}
$$

It was shown in I that

$$
\left|w^{(j)}(x)\right| \leqq C e^{-\delta \mid x_{N} !} \quad(\delta>0) \text { for }\left|x_{N}\right| \text { large. }
$$

(4.27) and (4.28) imply that

$$
\begin{aligned}
\left\|t P_{j} J U_{0}(t) \psi\right\|_{L_{2}(\Omega)}^{2} & =\left\|t w^{(j)}\left(J U_{0}(t) \psi, w^{(j)}\right)_{(\Omega)}\right\|_{L_{2}(\Omega)}^{2} \\
& =\left|\left(t J U_{0}(t) \psi, w^{(j)}\right)_{(\Omega)}\right|^{2} \\
& \leqq \int_{\Omega}\left|t U_{0}(t) \psi(x) w_{j}(x)\right|^{2} d x \\
& \leqq C .
\end{aligned}
$$

(4.26) and (4.29) together imply (4.25). Q.E.D.

Combining Lemma 4.2 with the proof of Theorem 4.1 , we easily see that this theorem remains true even if $A$ has eigenvalues.

5. The scattering operator. In this section we apply Theorem 4.1 to the problem of scattering in an infinite cylinder. We begin by establishing a local energy decay 
theorem for solutions $\mu^{0}(x, t)$ of the wave equation in $S$. Suppose $f=\left[f_{1}, f_{2}\right] \in \mathscr{H}_{0}$ and $\left[\mu^{0}(\cdot, t), \mu_{t}^{0}(\cdot, t)\right]=\mathscr{U}_{0}(t) f$.

LEMMA 5.1. If $B$ is an arbitrary bounded subset of $S$, then

$$
\int_{B}\left[\left|\nabla \mu^{0}(x, t)\right|^{2}+\left|\mu_{t}^{0}(x, t)\right|^{2}\right] d x \rightarrow 0 \text { as } t \rightarrow \pm \infty .
$$

Proof. Suppose $f_{j}(j=1,2)$ satisfies the hypothesis of Lemma 4.1. We may' use the spectral representation $\mathscr{T}^{0}$ and integrate by parts as in the proof of Lemma 4.2 to show that $\max _{B}\left|\mu^{0}(x, t)\right|=O\left(|t|^{-1}\right)$ and $\max _{B}\left|\mu_{t}^{0}(x, t)\right|=O\left(|t|^{-1}\right)$. (5.1) now follows for arbitrary elements $f$ in $\mathscr{H}_{0}$ by taking the limit in $\mathscr{H}_{0}$. Q.E.D.

Lemma 5.1 could also be proven by using the fact that the right hand side of (4.1) is independent of the "cutoff" function $\sigma(x)$. We now define the scattering operator $\mathscr{S}$ as follows:

$$
\mathscr{S} f=\left(\mathscr{W}^{+}\right)^{-1} \mathscr{W}^{-} f \quad \text { for } f \text { in } \mathscr{H}_{0} .
$$

It follows from Theorem 4.1 that $\mathscr{S}$ is a unitary transformation from $\mathscr{H}_{0}$ onto $\mathscr{H}_{0}$. Another characterization of $\mathscr{S}$ is given by the following theorem. (Again for simplicity we assume that $A$ has no point eigenvalues.) Note that we are extending the definition of the norm \|\|$_{\mathscr{H}}$ to all elements $h=\left[h_{1}, h_{2}\right] \in \mathscr{H}_{0}$ by setting

$$
\|h\|_{\mathscr{H}}^{2}=\int_{\Omega}\left[\left|\nabla h_{1}(x)\right|^{2}+\left|h_{2}(x)\right|^{2}\right] d x .
$$

THEOREM 5.1. Given arbitrary initial data $g$ in $\mathscr{H}$, there exists initial data $f=\left[f_{1}, f_{2}\right]$ in $\mathscr{H}_{0}$ such that

$$
\left\|\mathscr{U}(t) g-\mathscr{U}_{0}(t) f\right\|_{\mathscr{H}} \rightarrow 0 \quad \text { as } t \rightarrow-\infty,
$$

and

$$
\left\|\mathscr{U}(t) g-\mathscr{S} \mathscr{U}_{0}(t) f\right\|_{\mathscr{H}} \rightarrow 0 \quad \text { as } t \rightarrow+\infty .
$$

Proof. Set $f=\left(\mathscr{W}^{-}\right)^{-1} g$. Thus $\mathscr{U}(t) g=\mathscr{U}(t) \mathscr{W}^{-} f$. Using the definition of $\mathscr{W}^{-}$, the unitarity of $\mathscr{U}(t)$, and (5.1) we see that

$$
\lim _{t \rightarrow \pm \infty}\left\|\mathscr{U}(t) \mathscr{W}^{ \pm} f-\mathscr{U}_{0}(t) f\right\|_{\mathscr{H}}=0
$$

This implies (5.3). Furthermore

Hence

$$
\mathscr{U}(s) \mathscr{W}^{ \pm}=s-\lim _{t \rightarrow \pm \infty} \mathscr{U}(s-t) \mathscr{J} \mathscr{U}_{0}(t-s) \mathscr{U}_{0}(s)=\mathscr{W}^{ \pm} \mathscr{U}_{0}(s) .
$$

$$
\begin{aligned}
\mathscr{S} \mathscr{U}_{0}(t) & =\left(\mathscr{W}^{+}\right)^{-1} \mathscr{W}^{-} \mathscr{U}_{0}(t)=\left(\mathscr{W}^{+}\right)^{-1} \mathscr{U}(t) \mathscr{W}^{-} \\
& =\mathscr{U}_{0}(t)\left(\mathscr{W}^{+}\right)^{-1} \mathscr{W}^{-}=\mathscr{U}_{0}(t) \mathscr{S} .
\end{aligned}
$$

An application of (5.2), (5.5), and (5.6) gives

$$
0=\lim _{t \rightarrow \infty}\left\|\mathscr{U}(t) \mathscr{W}^{+}(\mathscr{S} f)-\mathscr{U}_{0}(t)(\mathscr{S} f)\right\|_{\mathscr{H}}=\lim _{t \rightarrow \infty}\left\|\mathscr{U}(t) \mathscr{W}^{-} f-\mathscr{S} \mathscr{U}_{0}(t) f\right\|_{\mathscr{H}}
$$

(5.7) clearly implies (5.4). Q.E.D. 
Finally we note that the infinite cylinder $S$ may be replaced by any perturbed cylinder $\Omega^{\prime}$ satisfying the conditions

(a) $\Omega \subseteq \Omega^{\prime} \subseteq S$,

(b) $\dot{\Omega}^{\prime}$ is a $C^{\infty}$ surface, and

(c) $A_{\Omega^{\prime}}$ has no point eigenvalues $\left({ }^{3}\right)$.

It may be shown that all of the results of $\mathrm{I}$ and this paper hold for the operators $A_{\Omega^{\prime}}$ and $A_{\Omega}=A$. The proofs are essentially unchanged.

6. Invariance of the wave operators. In this section we establish the existence in $L_{2}(\Omega)$ of the wave operators

$$
W^{ \pm}=W^{ \pm}\left(\varphi\left(A^{1 / 2}\right), \varphi\left(A_{0}^{1 / 2}\right)\right)=s-\lim _{t \rightarrow \pm \infty} W(t)
$$

where

$$
W(t)=\exp \left[-i \varphi\left(A^{1 / 2}\right) t\right] J \exp \left[i \varphi\left(A_{0}^{1 / 2}\right) t\right] .
$$

The functions $\varphi(\lambda)$ we shall consider satisfy the following conditions:

(i) $\varphi(\lambda)$ is a real-valued function defined on $\left(\nu_{1}, \infty\right)$,

(ii) the interval $\left(\nu_{1}, \infty\right)$ may be partitioned into subintervals $I_{\kappa}=\left(\lambda_{\kappa-1}, \lambda_{\kappa}\right)$, (i.e. $\left(\nu_{1}, \infty\right)=\bigcup_{\kappa=-\infty}^{\infty} I_{\kappa}$ ), where $\lambda_{\kappa} \rightarrow \nu_{1}$ as $\kappa \rightarrow-\infty, \lambda_{\kappa} \rightarrow \infty$ as $\kappa \rightarrow \infty$, and $\varphi(\lambda) \in C^{4}$ for $\lambda$ in $I_{\kappa}$. Also $\varphi^{\prime}(\lambda) \neq 0$ for $\lambda$ in $I_{\kappa}$.

Set $U_{0}(t)=\exp \left[i \varphi\left(A_{0}^{1 / 2}\right) t\right]$ and $U(t)=\exp \left[i \varphi\left(A^{1 / 2}\right) t\right]$. Let $P$ denote the projection onto the space spanned by the eigenfunctions of $A$.

THEOREM 6.1. (a) $W^{ \pm}=s-\lim _{t \rightarrow \pm \infty} W(t)$ are unitary transformations from $L_{2}(S)$ onto $(I-P) L_{2}(\Omega)$.

(b) Suppose $f \in L_{2}(S)$. Then if $\varphi^{\prime}(\lambda)>0$ on $I_{\kappa}$,

$$
\left(W^{ \pm} f\right)_{n}^{ \pm}(\xi)=\hat{f}_{n}^{0}(\xi) \quad \text { for } \xi^{2}+\nu_{n} \text { in } I_{\kappa},
$$

and if $\varphi^{\prime}(\lambda)<0$ on $I_{\kappa}$,

$$
\left(W^{ \pm} f\right)_{n}^{\mp \wedge}(\xi)=\hat{f}_{n}^{0}(\xi) \quad \text { for } \xi^{2}+\nu_{n} \text { in } I_{\kappa} .
$$

The proof of this theorem follows along the same lines as the proof of Theorem 4.1 with $\lambda$ replaced by $\varphi(\lambda)$ and therefore will not be given. Note that the function $U(t) f=\mu(x, t)$ satisfies the initial value problem

$$
\begin{aligned}
& \mu_{t}(x, t)=i \varphi\left(A^{1 / 2}\right) \mu(x, t) \quad \text { in } \Omega, \\
& \mu(x, 0)=f(x) .
\end{aligned}
$$

We may construct the scattering operator associated with equation (6.1) in the same manner as in $\$ 5$.

${ }^{3}$ Condition (c) may be dispensed with by considering only the continuous part of the operator $A_{\mathbf{\Omega}^{\prime}}$. 
All of the results of this paper hold for semi-infinite cylinders as well as for the boundary conditions discussed in I. The proofs remain the same.

Acknowledgement. The author wishes to express his sincere thanks to Professor P. D. Lax for valuable discussions concerning this and related problems.

\section{BIBLIOGRAPHY}

1. C. Goldstein, Eigenfunction expansions associated with the Laplacian for certain domains with infinite boundaries. I, Trans. Amer. Math. Soc. 135 (1969), 1-31.

2. T. Ikebe, Eigenfunction expansions associated with the Schroedinger operator and their applications to scattering theory, Arch. Rational Mech. Anal. 5 (1960), 1-34.

3. T. Kato, Wave operators and unitary equivalence, Pacific J. Math. 15 (1965), 171-174.

4. P. D. Lax and R. S. Phillips, Scattering theory, Academic Press, New York, 1967.

5. N. Shenk, Eigenfunction expansions and scattering theory for the wave equation in an exterior region, Arch. Rational Mech. Anal. 21 (1966), 120-150.

6. - The invariance of the wave operators associated with perturbations of $-\Delta$, J. Math. Mech. 17 (1968), 1005-1021.

BrookHaVen NATIONAL Laboratory, LONG IsLAND, NEW YoRK

NeW York UNIVERSITY, New York, New York 